\title{
Insights into the Distinguishing Stress-induced Cytotoxicity of Chiral Gold Nanoclusters and the Relationship with GSTPI
}

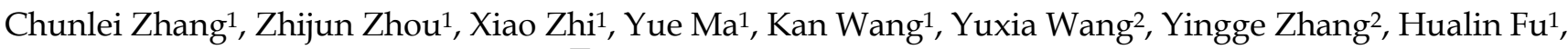 \\ Weilin Jin ${ }^{1}$, Fei Pan $^{1}$ and Daxiang Cui ${ }^{\bowtie}$ \\ 1. Institute of Nano Biomedicine and Engineering, Key Laboratory for Thin Film and Microfabrication Technology of the Ministry of Ed- \\ ucation, Department of Instrument Science \& Engineering, School of Electronic, Information and Electrical Engineering, Bio-X Center, \\ Institute of Translation Medicine, Shanghai Jiao Tong University, 800 Dongchuan Road, Shanghai 200240, China; \\ 2. Institute of Pharmacology and Toxicology, Academy of Military Medical Sciences, 47 Taping Road, Peking 100850, PR China.
}

$\square$ Corresponding author: Daxiang Cui, E-mail: dxcui@sjtu.edu.cn; Tel\&Fax: +86 021-34206886.

(c) Ivyspring International Publisher. This is an open-access article distributed under the terms of the Creative Commons License (http://creativecommons.org/ licenses/by-nc-nd/3.0/). Reproduction is permitted for personal, noncommercial use, provided that the article is in whole, unmodified, and properly cited.

Received: 2014.08.18; Accepted: 2014.09.15; Published: 2015.01.0I

\begin{abstract}
Chiral gold nanoclusters (Au NCs) exhibit attracting properties owing to their unique physical and chemical properties. Herein we report for the first time chiral gold nanoclusters' cytotoxicity and potential molecular mechanism. The L-glutathione (i.e. L-GSH) and D-glutathione (i.e. D-GSH)-capped Au NCs were prepared and characterized by HRTEM, UV-vis, photoluminescence and circular dichroism (CD) spectroscopy. Results showed that the CD spectra of L-glutathione (i.e. L-GSH) and D-glutathione (i.e. D-GSH)-capped Au NCs exhibited multiple bands which were identically mirror-imaged, demonstrating that the chirality of GSH-capped NCs had contributions from both the metal core and the ligand. The effects of AuNCs@L-GSH and AuNCs@D-GSH on cells were similar based on the cell physiology related cytotoxicity, although the effects became more prominent in AuNCs@D-GSH treated cells, including ROS generation, mitochondrial membrane depolarization, cell cycle arrest and apoptosis. Global gene expression and pathway analysis displayed that both AuNCs@L-GSH and AuNCs@D-GSH caused the up-regulation of genes involved in cellular rescue and stress response, while AuNCs@D-GSH individually induced up-regulation of transcripts involved in some metabolic- and biosynthetic-related response. MGC-803 cells were more sensitive to the oxidative stress damage induced by chiral Au NCs than GES-I cells, which was associated with GSTPI hypermethylation. In conclusion, chiral gold nanoclusters exhibit this chirality-associated regulation of cytotoxicity, different gene expression profiling and epigenetic changes should be responsible for observed phenomena. Our study highlights the importance of the interplays between chiral materials and biological system at sub-nano level.
\end{abstract}

Key words: gold nanocluster; chirality; cytotoxicity; ROS; methylation; epigenetic analysis.

\section{Introduction}

Chirality is one of inspiring and amazing phenomena in nature [1]. For example, the central components in life such as proteins and nucleic acids are chiral. All other amino acids excluding glycine in proteins are the L-enantiomers, while nucleic acids consisting of chains of deoxyribonucleosides (for DNA) or ribonucleosides (for RNA), are based on D-deoxyribose or D-ribose sugar ring, respectively [2, 3]. Up to date, chirality has inspired great advances in physics, chemistry, and life science, also has been 
found in a variety of gold nanoparticles (Au NPs) and gold nanoclusters (Au NCs) [3-5]. The field of fluorescent $\mathrm{Au}$ NCs has spurred much interest in recent years. The sub-nano sized Au NCs fill the "missing gap" between atomic and nanoparticle structures and exhibit molecular-like properties including discrete electronic states and size-dependent fluorescence, which are different from their larger counterparts-plasmonic nanocrystals [6,7]. Especially, chiral gold nanoclusters, as an emerging multifunctional nanomaterial, own great potential applications in areas such as sensing, catalysis, and biomedicine $[3,8$, 9].

Chiral optically activity of Au NCs was firstly observed by Schaaff and Whetten in 1998 [10]. A pair of $\mathrm{Au}$ NC enantiomers was also prepared by using optically active thiols such as D-and L-penicillamine [11]. Among the thiolate protected Au NCs, chiral L-glutathione (i.e. L-GSH; $N$ - $\gamma$-glutamyl-cysteinylglycine), a naturally occurring and readily available tripeptide, which intensively exists in living cells to maintain the cellular potential in a reducing state and to protect biological molecules against oxidative damage, was mainly used as the thiolate ligand for the synthesis of Au NCs [3,12]. Jin has explicitly revealed that -SG ligands and surface of gold atoms play a critical role in effecting the circular dichroism responses in metal-based electronic transitions [5,7]. Recently, Bürgi' group has reported the racemization of a chiral cluster, which indicates an intramolecular, concerted rearrangement process and the flexibility of the gold-thiolate interface [13]. The interfacial bonding structures seriously affect the properties of $\mathrm{Au}$ NCs [14]. It is evident that the ligand shell can tune the (bio) chemical properties and exert a tremendous influence on the applications of thiolate-protected gold nanoclusters. Therefore, there is a critical need for a systematic evaluation and understanding of chiral nanoclusters-dependent response in biological systems. Moreover, a better understanding of the interactions between chiral Au NCs and cells is helpful to clarify the mechanism of bioactivity of the Au NCs, which may lay foundation for the safe use of chiral nanomaterials. By far, several reports have shown that $\mathrm{Au}$ NCs are good biocompatible, can be excreted from kidney, and own great potential in applications such as in vivo fluorescent imaging- and/or X-ray CT imaging-guided chemotherapy and radiotherapy, which highlights the distinctive features of Au NCs of efficient renal clearance and enhanced passively tumor targeting capability [15-18]. However, currently, nanotoxicity of chiral Au NCs has not been clarified well, no report is closely associated with the influence of chirality of chiral Au NCs on human cell growth and proliferation, especially, no studies have focused on the biological process/molecular pathways on a gene expression based approach.

Herein, we prepared chiral Au NCs capped with enantiomers of L-GSH or D-GSH ( $N$ - $\gamma$-D-glutamylD-cysteinyl-glycine), and investigated their effects on human gastric cancer cell line MGC-803 and human gastric mucous epithelial cell line GES-1. Our study showed that both AuNCs@L-GSH and AuNCs@D-GSH can induce dose-dependent cytotoxicity for both MGC-803 and GES-1 cells, and cell physiology-based toxicity study revealed the integrated cytotoxic effects including ROS generation, mitochondrial depolarization, DNA damage, cell cycle arrest, and apoptosis mediated death. But D-GSH-capped Au NCs are obviously more toxic than L-GSH-capped ones for both MGC-803 and GES-1 cells; this chirality-associated regulation of cytotoxicity highlights the importance of the interplays between chiral materials and biological system at sub-nano level. DNA microarrays were used to investigate and compare the global gene expressions of MGC-803 cells treated with AuNCs@L-GSH and AuNCs@D-GSH. Both L-and D-GSH-capped Au NCs caused the up-regulation of a number of genes involved in cellular rescue and stress response, while AuNCs@D-GSH individually induced up-regulation of transcripts involved in some metabolic- and biosynthetic-related response. By virtue of the analysis of global gene expression in cells allowed us to obtain comprehensive knowledge of the cytotoxic effects of AuNCs@L-GSH and AuNCs@D-GSH. Another surprising finding was that whether co-incubating with AuNCs@L-GSH or AuNCs@D-GSH, the viability of MGC-803 cells was distinctly lower than the GES-1 cells. Taken together, the research on the interplay between chiral Au NCs and live cells will be helpful to explore the application prospects of chiral Au NCs in clinical theranostics.

\section{Materials and methods}

\section{Materials}

Gold (III) chloride trihydrate $\left(\mathrm{HAuCl}_{4} \bullet 3 \mathrm{H}_{2} \mathrm{O}\right.$, 99\%) was obtained from Sinopharm Chemical Reagent Co., Ltd, China. Dimethyl sulfoxide (DMSO) and glutathione (L-GSH) were obtained from Amresco (Solon, OH, USA). D-GSH (98\%) was synthesized by GL Biochem (Shanghai, China). Reactive Oxygen Species Assay Kit, GSH and GSSG Assay Kit, BCA (bicinchoninic acid) protein assay Kit and Mitochondrial membrane potential assay kit with JC-1were obtained from the Beyotime Institute of Biotechnology (Jiangsu, China). 5-Aza-2'-deoxycytidine (5-aza-dC; 98\%) and Tetrabutylammonium borohydride (TBAB; 95\%) were purchased from Aladdin Reagent Co. Ltd. 
(Shanghai, China). Trizol, 3-[4,5-dimethylthiazol2yl]-2, 5-diphenyltetrazolium bromide (MTT) and 2',7'-dichlorofluorescein diacetate (DCFH-DA) were purchased from Invitrogen Corporation (Carlsbad, CA, USA). Annexin V-FITC/PI Apoptosis Detection Kit was purchased from Yeasen Corporation (Shanghai, China). Direct-zol ${ }^{\mathrm{TM}}$ RNA MiniPrep and EZ DNA Methylation-Gold kit were purchased from Zymo Research (Orange, CA). Random primer and M-MLV reverse transcriptase were purchased from Promega (Madison, WI, USA). Anti-GSTP1 antibody was purchased from Bioss (Beijing, China); anti- $\beta$-actin antibody was purchased from AbMart (Shanghai, China). BM Chemiluminescence Western Blotting kit was purchased from Roche (Roche Molecular Biochemicals, Indianapolis, IN). MGC-803, GES-1 and MCF-7 cell lines were available in the Cell Bank of Type Culture Collection of Chinese Academy of Sciences. Cell culture products and reagent, unless mentioned otherwise, were purchased from GIBCO. All the above chemicals were used without any further purification. Ultrapure water (Millipore Milli-Q grade) with a resistivity of $18.2 \mathrm{M} \Omega \mathrm{cm}$ was used in all the preparations.

\section{Synthesis of fluorescent GSH-capped Au NCs}

The synthesis of red-emitting of L-GSH and D-GSH-capped Au NCs was done according to our previous procedure [6]. In a typical synthesis, freshly prepared aqueous solutions of $\mathrm{HAuCl}_{4}(10 \mathrm{mM}, 10$ $\mathrm{ml}$ ) and GSH (L-GSH or D-GSH; $150 \mathrm{mM}, 2 \mathrm{ml}$ ) were mixed and stirred for $20 \mathrm{~min}$ at room temperature. The reaction mixture was then cooled down to $0{ }^{\circ} \mathrm{C}$ (ice-bath), and subsequently, tetrabutylammonium borohydride (5 equiv/ $\mathrm{mol}$ of $\mathrm{Au}, 3 \mathrm{ml}$ ) was added rapidly and then stirred quickly for $4 \mathrm{~h}$. A side-product of the insoluble substance was identified to be $\mathrm{Au}(\mathrm{I})-\mathrm{SG}$ complexes [6]. The supernatant was further purified by adding three times amount of ethanol into the aqueous solution and centrifuge at 10,000 rpm for $15 \mathrm{~min}$. Under such condition, the Au NCs were precipitated out of the solution while the free GSH and gold ions remained in the solution. The precipitates were then re-suspended in ultrapure water and stored at $4{ }^{\circ} \mathrm{C}$ for further experiments.

\section{Characterization of Au NCs}

The sizes and morphologies of the Au NCs were characterized by High-resolution TEM (HRTEM) on JEM-2100F (JEOL, Japan) with an acceleration voltage of $200 \mathrm{kV}$. UV-vis spectra were measured with a Varian Cary 50 spectrophotometer (Varian Inc., Palo Alto, CA, USA) equipped with a $10-\mathrm{mm}$ quartz cell, where the light path length was $1 \mathrm{~cm}$. Photoluminescence (PL) and photoluminescence excitation (PLE) spectra of $\mathrm{Au}$ NCs were recorded on a Hitachi FL-4600 spectrofluorometer. Circular dichroism (CD) measurements of Au NCs were performed on a Jasco J-815 CD spectrometer (Jasco International, Tokyo, Japan) in quartz cuvettes with a path length of $10 \mathrm{~mm}$ at ambient temperature. Data were collected every $0.2 \mathrm{~nm}$ with a bandwidth of $1 \mathrm{~nm}$, at $50 \mathrm{~nm} \mathrm{~min}{ }^{-1}$ and averaging over three scans. Size distribution and zeta potential of $\mathrm{Au}$ NCs were determined by dynamic light scattering using a NICOMP 380 ZLS Zeta potential/Particle sizer (PSS Nicomp, Santa Barbara, CA, USA).

\section{Cell culture and cell viability assay}

The human gastric cancer cell line MGC-803, human gastric epithelial cell line GES-1, and human breast adenocarcinoma cell line MCF-7 were maintained at $37{ }^{\circ} \mathrm{C}\left(5 \% \mathrm{CO}_{2}\right)$ in Dulbecco's Modified Eagle's Medium (DMEM, HyClone) supplemented with $10 \%$ (vol/vol) fetal bovine serum (Gibco), $100 \mathrm{U} / \mathrm{mL}$ penicillin and $1 \mathrm{mg} / \mathrm{mL}$ streptomycin. Cell viability was determined by the 3-[4,5-dimethylthiazol2yl]-2,5-diphenyltetrazolium bromide (MTT) assay. Briefly, cells were seeded at a density of $5 \times 10^{3}$ cells/well in a 96-well plate (Corning, Costar, NY) with $10 \%$ fetal bovine serum, and cultured overnight. The cells were incubated with various concentrations of $\mathrm{Au} \mathrm{NCs}$ for $24 \mathrm{~h}$. After exposure, the supernatant was removed and cells were washed once with phosphate-buffered saline (PBS) (pH 7.4).Then,150 $\mu \mathrm{l}$ DMEM and $15 \mu \mathrm{l}$ MTT $(0.5 \mathrm{mg} / \mathrm{ml})$ were added to each well and incubated for $4 \mathrm{~h}$ at $37^{\circ} \mathrm{C}$. Finally, $150 \mu \mathrm{l}$ DMSO was added to dissolve the formazan crystals. The absorbance was measured at $570 \mathrm{~nm}$ using a standard micro plate reader (Scientific Multiskan MK3, thermo, USA). The following formula was used: cell viability $=(\mathrm{OD}$ of the experimental sample $/ \mathrm{OD}$ of the control group) $\times 100 \%$ and the cell viability of control group were denoted as $100 \%$.

\section{Detection of Reactive Oxygen Species (ROS)}

Cellular generation of ROS in MGC-803 cells after exposure to $\mathrm{Au}$ NCs was measured using DCFH-DA. DCFH-DA is a nonfluorescent cell-permeable indicator for ROS that becomes fluorescent upon oxidation by ROS after cleavage of the protecting acetate groups by intracellular esterases. We used $0.3 \% \mathrm{H}_{2} \mathrm{O}_{2}$ as a positive control. Upon treatment with $0.3 \% \mathrm{H}_{2} \mathrm{O}_{2}$ stronger fluorescence indicated the cells suffered strong oxidative stress. After incubation with the Au NCs, the cell culture medium was replaced with serum free medium in the presence of $20 \mu \mathrm{M}$ of DCFH-DA. After $20 \mathrm{~min}$ incubation at 37 ${ }^{\circ} \mathrm{C}$, the dye was washed twice with serum free medium, and intracellular fluorescence was detected 
using fluorescence microscope. The fluorescence pictures were captured with Nikon ECLIPSE TS100 microscope, equipped with a $50 \mathrm{~W}$ mercury lamp and Nikon DS-Fil camera, NIS-Elements software was used for image acquisition. The fluorescence filter cube was the Nikon B-2A set (Excitation Filter Wavelengths: 450-490 $\mathrm{nm}$ bandpass, $470 \mathrm{CWL}$ ), Dichromatic Mirror Cut-on Wavelength: $500 \mathrm{~nm}$ (longpass, LP), Barrier Filter Wavelengths: 515 nanometer cut-on (longpass, LP). The objective CFI Plan Apochromat 20 $\times$ (NA 0.75, air correction) was used.

\section{GSH and GSSG Assay}

MGC-803 and GES-1 cells were seeded in 6-well plates at $1 \times 10^{6}$ cells per well. After $24 \mathrm{~h}$ of incubation with different concentration of $\mathrm{Au} \mathrm{NCs}$, cells were washed twice with PBS. Cells were digested and collected in a centrifuge tube and immediately add 3 volumes of ice-cold 5\% metaphosphoric acid, then frozen and thawed twice using liquid nitrogen and 37 ${ }^{\circ} \mathrm{C}$ water. The samples were centrifuged at $12000 \mathrm{rpm}$ for 10 minutes at $4{ }^{\circ} \mathrm{C}$ and the supernatant was used for GSH and GSSG assays. Total glutathione (GSH+GSSG), reduced glutathione (GSH) and oxidized glutathione (GSSG) were determined with the GSH and GSSG Assay Kit (Beyotime Biotech, Jiangsu, China) according to the protocol. The amount of reduced GSH was obtained by subtracting the amount of GSSG from that of total GSH.

\section{Mitochondrial membrane potential $\left(\Delta \Psi_{\mathrm{m}}\right)$ detection}

Fluorescence microscope was used for the analysis of MMP. Briefly, after Au NCs treatment, the cells were incubated with $5 \mathrm{mg} / \mathrm{L} \mathrm{JC}-1$ (Beyotime Biotech) at $37^{\circ} \mathrm{C}$ for $20 \mathrm{~min}$ and rinsed twice with ice-cold PBS. Mitochondrial membrane potentials were monitored by determining the relative amounts or dual emissions from both mitochondrial JC-1 monomers and aggregates using a Nikon ECLIPSE TS100 microscope. The Fluorescence filter cube was Nikon B-2A set as mentioned above. NIS-Elements software was used for image acquisition. The objective CFI Plan Apochromat $20 \times$ (NA 0.75 , air correction) was used.

\section{DNA damage assay}

Detection of cellular $\gamma \mathrm{H} 2 \mathrm{AX}$ was carried out by using the $\mathrm{H} 2 \mathrm{AX}$ phosphorylation assay kit for flow cytometry (Millipore, USA). MGC-803 cells were treated with $100 \mu \mathrm{g} / \mathrm{ml}$ AuNCs@L-GSH and AuNCs@D-GSH for 24, respectively. GES-1 cells were treated under the same condition as MGC-803 cells. Cells only without any treatment were denoted as control. The assay was carried out according to the manufacturer's protocol with one major modification: the FITC-labeled antibody was incubated overnight at
$4{ }^{\circ} \mathrm{C}$ instead of $20 \mathrm{~min}$ on ice. The position along the $x$-axis correlates to the amount of $\gamma \mathrm{H} 2 \mathrm{AX}$ and any events under the right gate signify a positive signal-phosphorylated H2AX ( $\gamma \mathrm{H} 2 \mathrm{AX})$. The number in the upper right corner of each panel indicated the percentage of cells scoring positive for staining with the $\mathrm{\gamma H} 2 \mathrm{AX}$ antibody.

\section{Cell cycle analysis}

Cell cycle analysis was carried out by PI staining followed by flow cytometric measurement. In brief, cells were washed twice by PBS, and trypsinized, harvested and centrifuged after Au NCs treatment for $24 \mathrm{~h}$. Cell pellet was washed twice with cold PBS and fixed overnight in $70 \%$ ethanol at $-20^{\circ} \mathrm{C}$. Before flow cytometry analysis, cells were washed with PBS and stained with $50 \mu \mathrm{g} / \mathrm{ml}$ PI containing RNase A (100 $\mu \mathrm{g} / \mathrm{ml}$ ) and incubated at $37^{\circ} \mathrm{C}$ for $30 \mathrm{~min}$, followed by analyzed with a FACSCalibur (BD Biosciences, Mountain View, CA). Cell debris and clumps were gated out, and $G_{1}, S$, and $G_{2} / M$ populations were quantified using FlowJo 7.6 software.

\section{Apoptosis assay by Annexin V-FITC and propidium iodide ( $\mathrm{PI})$ double staining}

The apoptotic and necrotic cell distribution were analyzed by Annexin V/PI apoptosis detection Kit (Yeasen, Shanghai). MGC-803 and GES-1 cells were treated with $100 \mathrm{\mu g} / \mathrm{ml}$ AuNCs@L-GSH and AuNCs@D-GSH for $24 \mathrm{~h}$ respectively; cells were harvested, washed once with PBS and resuspended in $200 \mu \mathrm{l}$ binding buffer, $5 \mu \mathrm{l}$ of Annexin V-FITC and 10 $\mu \mathrm{l}$ of PI was added and incubated in the dark at room temperature for $15 \mathrm{~min}$. Then, $400 \mu \mathrm{l}$ of binding buffer was added to each sample, and the cells were immediately analyzed by flow cytometry. The data analysis was performed with FlowJo 7.6 software. Positioning of quadrants on Annexin V/PI plots was performed to distinguish intact (Annexin $\mathrm{V}^{-} / \mathrm{PI}^{-}$), early apoptotic (Annexin $\left.\mathrm{V}^{+} / \mathrm{PI}^{-}\right)$, late apoptotic (Annexin $\mathrm{V}^{+} / \mathrm{PI}^{+}$) and necrotic (Annexin $\mathrm{V}^{-} / \mathrm{PI}^{+}$) cells.

\section{Microarray and global gene expression analysis}

\section{Experiment}

MGC-803 cells cultured previously in DMEM for $24 \mathrm{~h}$ were further cultured for $24 \mathrm{~h}$ in media containing AuNCs@L-GSH (100 g/ml) and AuNCs@D-GSH $(100 \mu \mathrm{g} / \mathrm{ml})$, respectively. The cells cultured in the media only were denoted as control group. Then total RNA was extracted from treated and control cells and the RNAs integrity was assessed using Agilent Bioanalyzer 2100 (Agilent Technologies). The sample labeling, microarray hybridization and washing were performed based on the manufacturer's standard protocols. Briefly, total RNAs were transcribed to 
double strand cDNAs and then synthesized cRNAs. Next, 2nd cycle cDNAs were synthesized from cRNAs. Followed fragmentation and biotin labeling, the 2nd cycle cDNAs were hybridized onto the Affymetrix Human HTA 2.0 microarray. After washing and staining, the arrays were scanned by the Affymetrix Scanner 3000 (Affymetrix).

\section{Microarray data analysis}

Affymetrix GeneChip Command Console (version 4.0, Affymetrix) software was used to extract raw data. The data were subjected to normalization, background correction and summarization using Expression Console (version1.3.1, Affymetrix) software with the standard Robust Multi-array Average (RMA) algorithm. Genespring software (version 12.5; Agilent Technologies) was employed to finish the basic gene expression analysis. Differentially expressed genes were then identified through fold change. The threshold set for up- and down-regulated genes was a fold change $>=1.5$. Afterwards, GO analysis and KEGG analysis (analyzed using the online tool FunNet) were applied to determine the roles of these differentially expressed mRNAs played in these GO terms or pathways [46].

\section{RT-PCR}

Total RNA from MGC-803, GES-1, and MCF-7 cells was isolated using Trizol (Invitrogen) and Direct-zol $^{\text {TM }}$ RNA MiniPrep (Zymo Research) according to manufacturer's instructions. The RNA was quantified using a nanodrop ND-1000 (Thermo Fischer Scientific). First-strand cDNA was obtained by using $1 \mu \mathrm{g}$ of total RNA and random primers and M-MLV reverse transcriptase (Promega). $\beta$-actin was chosen as the endogenous control in the assay. The cDNA templates were stored at $-20^{\circ} \mathrm{C}$. The following PCR primers were used: $\beta$-actin primers, forward: 5'-GTGGGGCGCCCCAGGCACCA-3', reverse: 5'-CT CCTTAATGTCACGCACGATTTC-3'; GSTP1 primers, forward: 5'-GTAGTTTGCCCAAGGTCAAG-3', reverse: 5'-AGCCACCTGAGGGGTAAG-3'. The PCR protocol was: $3 \mathrm{~min}$ at $95^{\circ} \mathrm{C} ; 1 \mathrm{~min}$ at $94^{\circ} \mathrm{C}, 1 \mathrm{~min}$ at 60 ${ }^{\circ} \mathrm{C}, 1 \mathrm{~min}$ at $72{ }^{\circ} \mathrm{C}$ for 35 cycles; $72{ }^{\circ} \mathrm{C}, 10 \mathrm{~min}$. PCR products were separated on the $20 \mathrm{~g} / \mathrm{L}$ agarose gel stained with ethidium bromide and viewed under ultraviolet light.

\section{Methylation-specific PCR (MSP)}

Genomic DNA was isolated from the MGC-803, GES-1 and MCF-7 cells and the methylation status of GSTP1 was examined using methylation-specific PCR (MSP). Five hundred nanograms of each DNA sample was converted with EZ DNA Methylation-Gold kit (Zymo Research) under the following conditions: 98 ${ }^{\circ} \mathrm{C}$ for $10 \mathrm{~min}, 5$ cycles of $64^{\circ} \mathrm{C}$ for $30 \mathrm{~min}$ followed by
$4{ }^{\circ} \mathrm{C}$ for $2 \mathrm{~h}$. Converted DNA was purified according to manufacturer's instructions and $1 / 10$ th was used as template per amplification. Methylation-specific PCR was carried out as follows: 1 cycle at $95^{\circ} \mathrm{C}$ for $3 \mathrm{~min}$; 35 cycles at $94{ }^{\circ} \mathrm{C}$ for $30 \mathrm{~s}$, then 30 s at $58^{\circ} \mathrm{C}$, 30s at 72 ${ }^{\circ} \mathrm{C}$, with final extension for $10 \mathrm{~min}$ at $72{ }^{\circ} \mathrm{C}$. Primer sequences for the methylated GSTP1 amplification were 5'-TTCGGGGTGTAGCGGTCGTC-3' (forward) and 5'-GCCCCAATACTAAATCACGACG-3' (reverse), and primer sequences for the unmethylated GSTP1 amplification were 5'-GATGTTTGGGGTGTA GTGGTTGTT-3' (forward) and 5'-CCACCCCAATAC TAAATCACAACA-3' (reverse). PCR products were analyzed on $12 \%$ polyacrylamide gel electrophoresis, stained with ethidium bromide, and visualized under UV illumination.

\section{Western blotting}

In brief, the total cell lysates were prepared in high $\mathrm{KCl}$ lysis buffer $(10 \mathrm{mM}$ Tris- $\mathrm{HCl}, \mathrm{pH} 8.0,140$ $\mathrm{mM} \mathrm{NaCl}, 300 \mathrm{mM} \mathrm{KCl}, 1 \mathrm{mM}$ EDTA, 0.5\% Triton X-100 and $0.5 \%$ sodium deoxycholate) with complete protease inhibitor cocktail (Roche). The protein concentration was determined using BCA (bicinchoninic acid) protein assay kit (Beyotime Biotech, Jiangsu, China) and store at $-20^{\circ} \mathrm{C}$. Thirty micrograms of protein were separated by sodium dodecyl sulfate polyacrylamide gel electrophoresis (SDS-PAGE) and transferred to polyvinylidene fluoride membranes (Roche). The membranes were treated with 1\% blocking solution in TBS for $2 \mathrm{~h}$, the membranes were incubated with primary antibodies anti-GSTP1 (1:200; Bioss, Beijing, China), anti-a-tubulin (1:1000; AbMart, Shanghai, China), at $4{ }^{\circ} \mathrm{C}$ overnight. Then the membranes were washed and incubated with POD-labeled secondary antibodies (Roche). The immunolabeled proteins were detected by BM Chemiluminescence Western Blotting kit (Roche).

\section{Statistical analysis}

All experiments were performed in triplicate unless otherwise indicated. Data are expressed as mean values \pm standard deviation. Statistical differences were calculated by a two-tailed, unpaired Student's $t$-test at a significance level of $p<0.05$.

\section{Results and discussion \\ Chiral Au NCs characterization}

The synthesis of chiral $\mathrm{Au}$ NCs was modified from the method we have reported [6]. High-resolution transmission electron microscopy (HRTEM) images indicated that all Au NCs exhibit the same outstanding size uniformity and spherical shape (Fig. 1A; for size distribution, see Supplementary Material: Fig. S1). The insets in Fig. 1a suggested 
that the lattice fringes of $\mathrm{Au} \mathrm{NCs}$ were consistent with the metallic gold lattice spacing of $0.24 \mathrm{~nm}$. No surface plasmon absorption peaks were observed for AuNCs@L-GSH or AuNCs@D-GSH (Fig. 1B), which is a unique feature of ultrasmall Au NCs. Because of quantum confinement effects, Au NCs are too small to support surface plasmon resonance. Photoluminescence (PL) and photoluminescence excitation (PLE) spectra of L- and D-GSH-capped Au NCs were essentially the same (Fig. 1C). The physicochemical properties of the AuNCs@L-GSH and AuNCs@D-GSH were summarized in Supplementary Material: Table S1; they had in fact an inner consistency. Free L- and D-GSH molecules showed mirror-image circular dichroism (CD) spectra in the region of 200 to $250 \mathrm{~nm}$ (Fig. 2A). Compared with chiral GSH molecules, Land D-GSH-capped Au NCs were optically active and possessed almost identical mirror images of one another in the range of 200 to $500 \mathrm{~nm}$ (Fig. 2B), and insoluble $\mathrm{Au}(\mathrm{I}) \mathrm{SG}$ complex were in the region of 200 to $700 \mathrm{~nm}$ (Supplementary Material: Fig. S2). Interestingly, the signal of L- and D-GSH-capped Au NCs was reversed compared with that of the ligand. Up to date, no satisfactory mechanism has been given to explain such reversal CD signals after chiral molecules capped onto the gold surface $[3,19]$. But, it is clear that the chiral optically activities of ligand-capped nanoclusters are both from the metal atom arrangement and the ligand shell around it $[3,8]$.
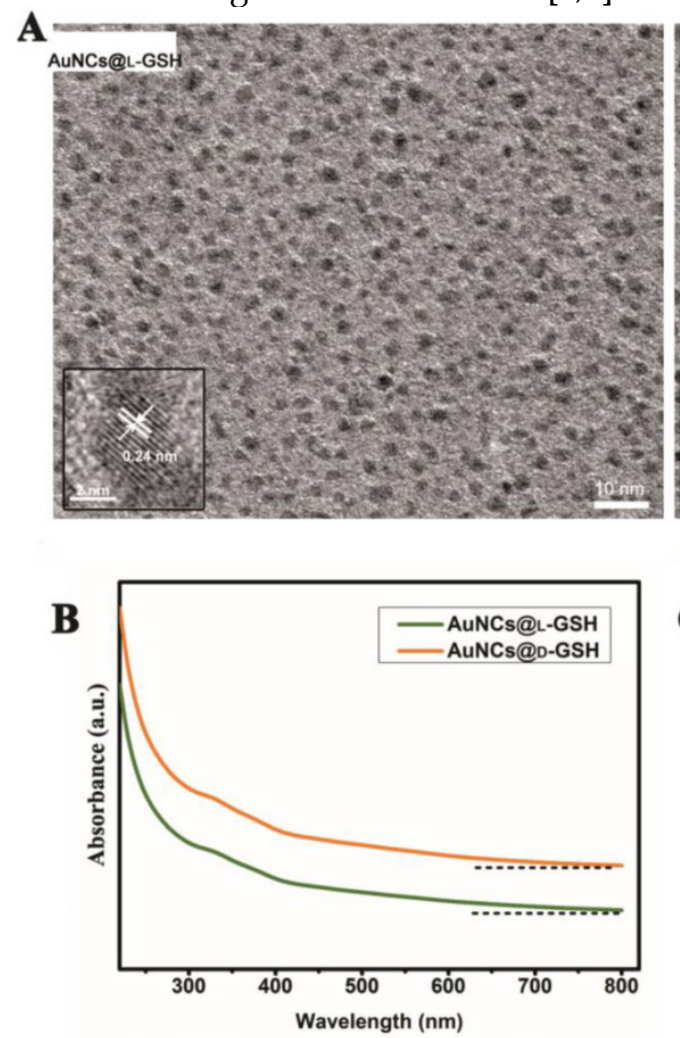

\section{Cytotoxicity and oxidative stress}

Cytotoxicity of L- and D-GSH-capped Au NCs was determined using the MTT colorimetric assay. AuNCs@L-GSH displayed little toxicity at dose up to $250 \mu \mathrm{g} / \mathrm{ml}$ against MGC-803 and GES-1 cells, with $\mathrm{IC}_{50}$ (half-maximum inhibitory concentration) values of $1068.656 \pm 59.241$ and $1183.871 \pm 71.254 \mu \mathrm{g} / \mathrm{ml}$, respectively (Fig. 3A and B). Conversely, AuNCs@D-GSH induced a sharp decrease in MGC-803 cell viability when the doses were up to 100 $\mu \mathrm{g} / \mathrm{ml}$. The $\mathrm{IC}_{50}$ values of MGC-803 and GES-1 cells induced by AuNCs@D-GSH were $87.336 \pm 3.365$ and $647.169 \pm 39.124 \mu \mathrm{g} / \mathrm{ml}$, respectively. In brief, our results clearly demonstrated that D-GSH-capped Au NCs were obviously more toxic than L-GSH-capped ones for both MGC-803 and GES-1 cells. We also investigated the bio-compatibility of free L- and D-GSH compounds. As shown in Supplementary Material: Fig. S3, none cytotoxicity was observed with neither L- nor D-GSH when the dose was up to $1 \mathrm{mg} / \mathrm{ml}$. Based on rigorous consideration of the stability of Land D-GSH-capped AuNCs, we tested the UV-vis absorption spectra of Au NCs in 0.01M PBS (pH, 7.4) and DMEM containing $10 \%$ FBS at $37{ }^{\circ} \mathrm{C}$ for $24 \mathrm{~h}$ (Supplementary Material: Fig. S4). The unchanged unique structure of L- and D-GSH-capped AuNCs was suggested by their almost overlapping optical absorption spectra.
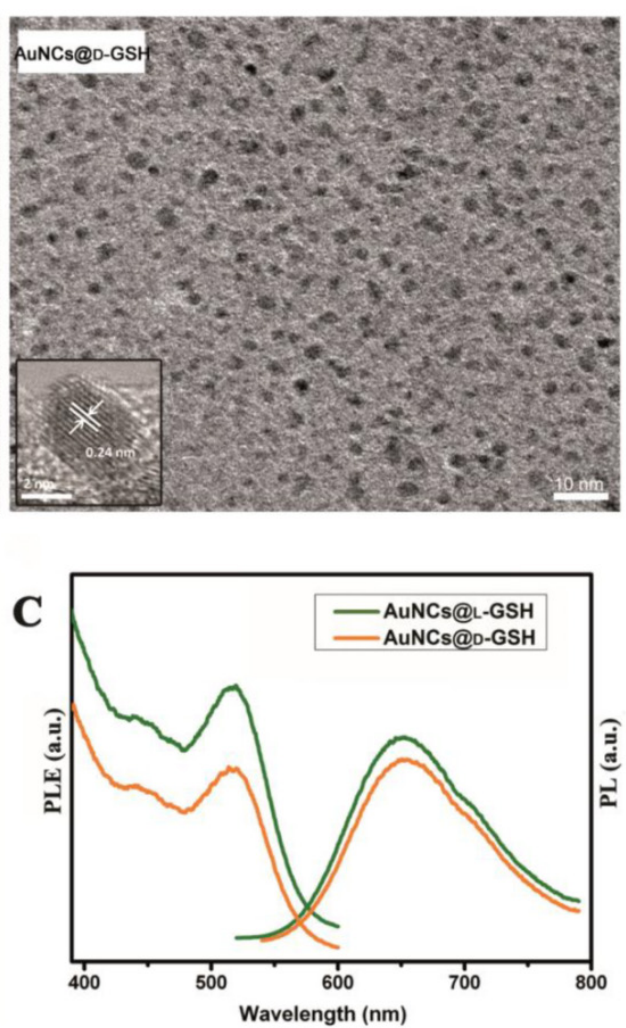

Figure I. Characterization of L- and D-GSH-capped Au NCs. (A) HRTEM images of AuNCs@L-GSH and AuNCs@D-GSH. The spherical Au NCs were marked with a distance of the lattice spacing (insets). (B) UV-vis spectra of AuNCs@L-GSH and AuNCs@D-GSH. (C) PL ( $\lambda_{\text {ex }}=520$ nm) and PLE ( $\lambda_{\text {em }}=650$ nm) spectra of L-and D-GSH-capped Au NCs. 
$\mathbf{A}$

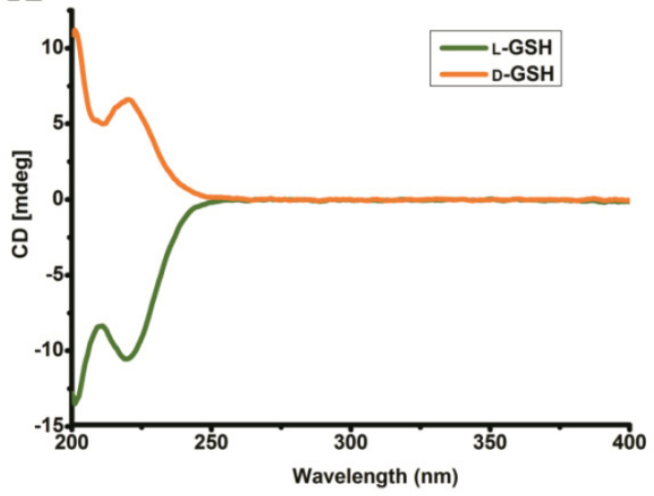

B

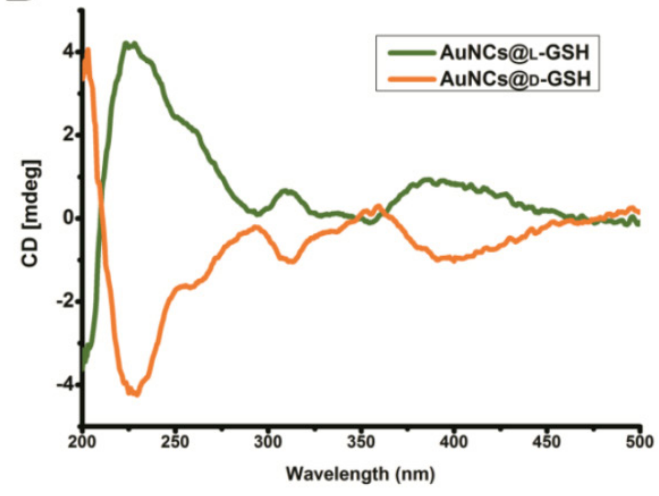

Figure 2. Circular dichroism spectra. (A) CD spectra of L- and D-GSH. (B) CD spectra of AuNCs@L-GSH and AuNCs@D-GSH.
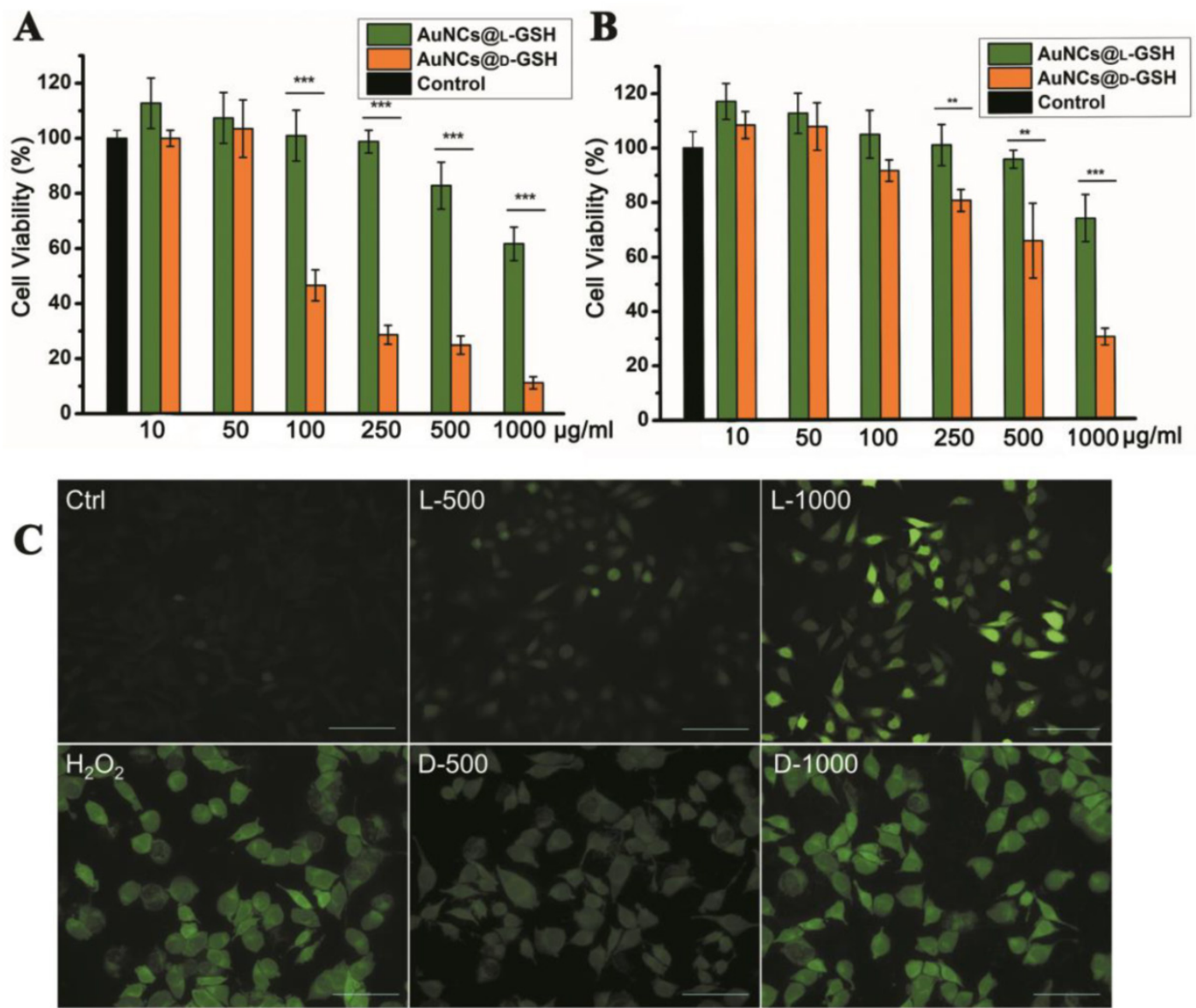

Figure 3. Cytotoxicity and oxidative stress. Concentration- and chirality-dependent cytotoxicity of Au NCs for (A) MGC-803 cells and (B) GES-Icells. (C) ROS level of MGC-803 cells after co-incubation with Au NCs for $12 \mathrm{~h}$ was examined by DCFH-DA under fluorescence microscope. L-500, L- I000, D-500, D- 1000 were denoted as MGC-803

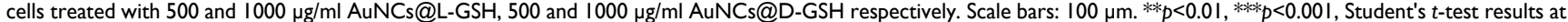
$95 \%$ confidence level were significantly different.

Regarding the mechanism of nanotoxicity, oxidative stress induction was a common paradigm for the cellular toxicity of nanoparticles. In order to establish the role of reactive oxygen species (ROS) was a decisive factor in chiral GSH-capped Au NCs cytotoxicity, 2',7'-dichlorofluorescein diacetate (DCFHDA) staining method was performed. DCFH-DA is a nonfluorescent cell-permeable indicator for ROS and becomes fluorescent upon oxidation by ROS after cleavage of the protecting acetate groups by intracellular esterases [20]. MGC-803 cells treated with $0.3 \%$ $\mathrm{H}_{2} \mathrm{O}_{2}$ for $0.5 \mathrm{~h}$ were used as positive control; media-only-treated cells were served as the negative control. As shown in Fig. 3C, a clearly concentration-and chirality-dependent induction of ROS was observed while the MGC-803 cells incubated with 
AuNCs@D-GSH produced more ROS than the cells incubated with AuNCs@L-GSH. GSH is a major component of the antioxidant defense system of mammalian cells, and it reduces ROS with the oxidation of the -SH group to convert to the dimer form of GSSG with S-S bond [21]. Changes of the intracellular thiol-disulfide (GSH/GSSG) balance can be used as an indicator of the redox status of the cell $[12,21]$. The intracellular GSH/GSSG levels were determined by the GSH and GSSG assay. Both AuNCs@L-GSH and AuNCs@D-GSH decreased the GSH/GSSG ratio of MGC-803 and GES-1 cells (Supplementary Material: Fig. S5). Next, we co-incubated AuNCs@D-GSH with L-GSH or D-GSH for the entire duration of the experiment. This treatment restored cell survival rate considerably in MGC-803 cells, while it was not statistically significant in GES-1 cells as shown in Supplementary Material: Fig. S6. Nevertheless, the D-GSH seems had the same ability to increase the cell viability as the biological active form L-GSH; this may be explained that thiol-containing compounds could neutralize the toxicity of ROS [22].

\section{Mitochondrial membrane potential}

In order to investigate the effects of chiral $\mathrm{Au}$ NCs on mitochondria, as the mitochondrion is ROS' main action site, the membrane-permeant JC-1 dye staining method is used to monitor mitochondrial status. Oxidative stress can decrease the mitochondrial membrane potential (MMP), ultimately leading to open the mitochondrial permeability transition pore, which results in a sudden increase in the permeability of the inner mitochondrial membrane [23].
In live cells, JC-1 exists either as an orange/red-fluorescent aggregates at hyperpolarized membrane potentials or as a green-fluorescent monomer at depolarized membrane potentials [24]. Therefore mitochondrial depolarization was expressed by an increase in the intensity ratio of green/red fluorescence. As illustrated in Fig. 4, untreated MGC-803 cells with polarized mitochondria appeared as red punctate staining while the MGC-803 cells treated with different concentrations of AuNCs@D-GSH and AuNCs@L-GSH caused progressive loss of red fluorescence and cytoplasmic diffusion of green fluorescence. The result of mitochondria potential staining of the GES-1cells was shown in Supplementary Material: Fig. S7. As demonstrated in Fig. 4 and Fig. S7, D-GSH-capped Au NCs could effectively increase the intensity ratio of green/red fluorescence than L-GSH-capped ones for a given concentration, which was fully compatiable with the results of ROS induction. All these results suggested that ROS generation induced by oxidative stress might be an important cytotoxicity mechanism for chiral Au NCs. What's more, the depolarization of mitochondrial membrane potential has been shown to be an early event in the apoptotic process and has even been suggested to be central to the apoptotic pathway $[23,25]$. To evaluate secondary effects on the context of cell physiology by the elevated ROS level and the depolarized of mitochondrial membrane potential, the analysis of DNA damage, cell cycle, and apoptosis was done.
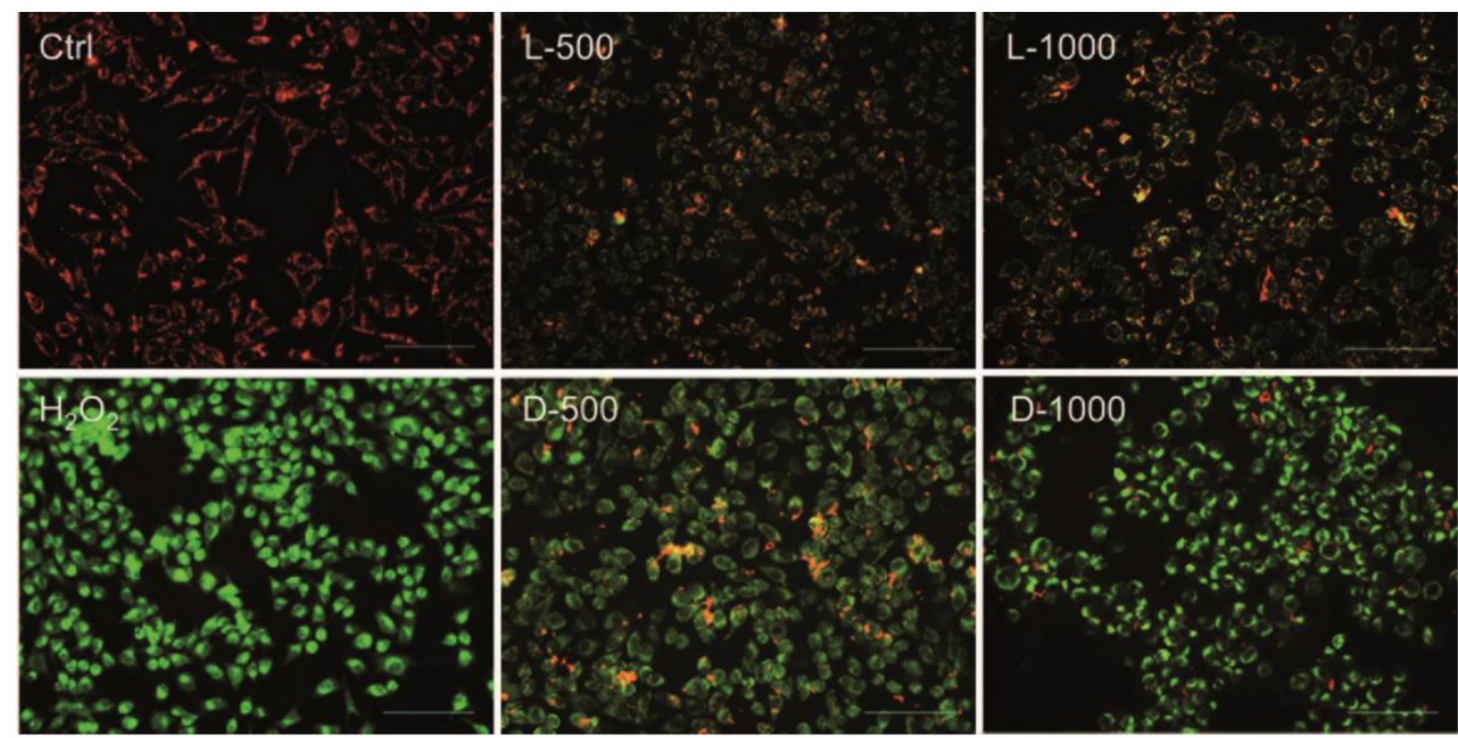

Figure 4. Fluorescent potential-dependent staining of mitochondria in MGC-803 cells by JC-I following exposure to Au NCs for I2 h. L-500, L-I000, D-500, D- I000 were denoted as MGC-803 cells treated with 500 and $1000 \mu \mathrm{g} / \mathrm{ml} \mathrm{AuNCs@L-GSH,} 500$ and $1000 \mu \mathrm{g} / \mathrm{ml}$ AuNCs@D-GSH, respectively. The cells were illuminated at $450-490$ nm and the emission was collected with a $515 \mathrm{~nm}$ longpass optical filter. Scale bars: $100 \mu \mathrm{m}$. 
$\mathbf{A}$

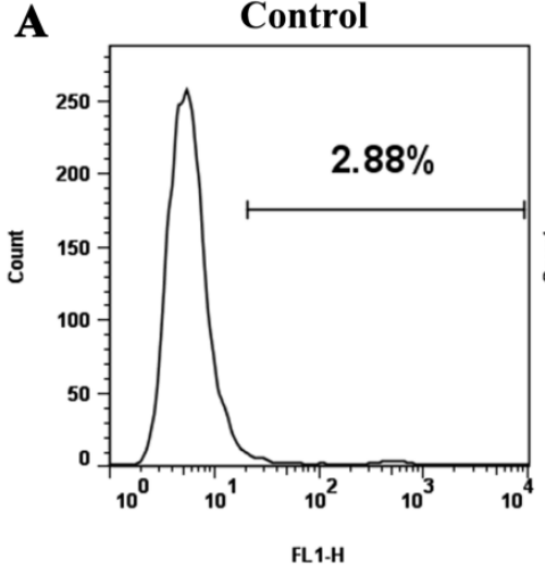

B

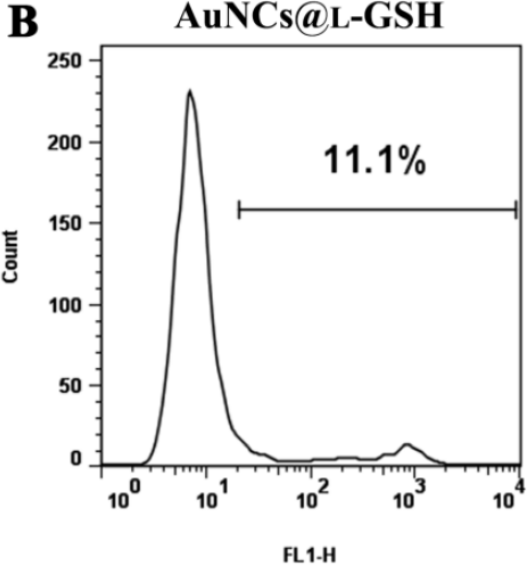

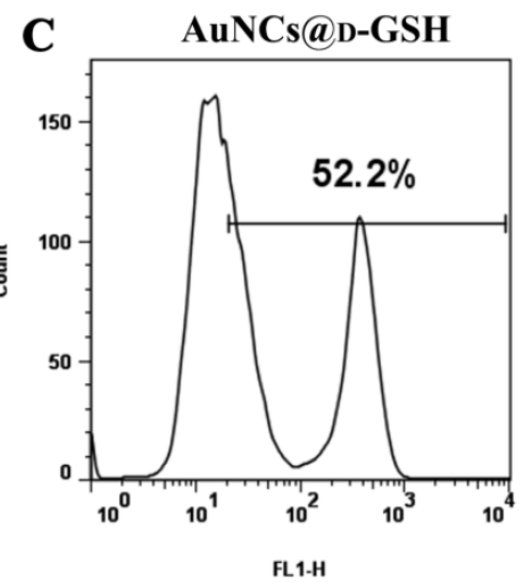

Figure 5. Flow cytometry analysis of the phosphorylation of H2AX. (A) MGC-803cells without any treatment. (B-C) MGC-803 cells treated with I00 $\mu g / m l$ AuNCs@L-GSH and AuNCs@L-GSH for 24, respectively. The phosphorylation of H2AX is assessed by the degree of shift of the cell population to the right. The number in the upper right corner of each panel indicates the percentage of cells scoring positive for staining with $\mathrm{yH} 2 \mathrm{AX}$ antibody.

\section{DNA damage assay}

The genotoxic potentiality of Au NCs was evaluated by measuring the activation of phosphorylated H2AX on Ser139 ( $\gamma \mathrm{H} 2 \mathrm{AX}$ ), a well-recognized protein biomarker for double-strand DNA breakage [26]. As shown in Fig. 5, both AuNCs@L-GSH and AuNCs@D-GSH induced a shift in the cellular status of $\gamma \mathrm{H} 2 \mathrm{AX}$ with activation peaks partitioned to the right while the percentage of cells scoring positive for H2AX phosphorylation induced by AuNCs@D-GSH was higher than that caused by AuNCs@L-GSH, which correlated well with our previous results of cytotoxicity and ROS generation. ROS-mediated genotoxicity has been previously observed for metal oxide nanoparticles [27]. This is the first investigation that provided quantitative measurements of distinguishing DNA damage in chiral Au NCs treated cells. The activation $\gamma \mathrm{H} 2 \mathrm{AX}$ was also found in GES-1 cells when treated with AuNCs@L-GSH and AuNCs@D-GSH (Supplementary Material: Fig. S8). Since the DNA damage is always with the downstream events that lead to recruitment of DNA repair machinery and engagement of cell cycle checkpoints, so the cell cycle distribution was examined [28].

\section{Cell cycle and apoptosis assay}

Recently, it has been reported that ROS influence cell cycle progression in a context-dependent manner via phosphorylation and ubiquitination of CDKs (cyclin dependent kinases) and other cell cycle regulatory molecules[29]. For example, ortho-quinonoid compound LGH00031 inhibited the activity of Cdc25b, thereby inhibiting the $\mathrm{G}_{2} / \mathrm{M}$ phase transition and decreasing tumor cell proliferation depending on the presence of ROS [30]. Moreover, damaged DNA elicited the activation of cell cycle $G_{2}$ checkpoints function to repair DNA damage before entering mi- tosis. As already explained in the previous DNA damage study, Au NCs treated MGC-803 cells indicated the breakage of double-strand DNA where the early effect will be evidenced in cell cycle progression. Then the toxicity studies were further extended to cell cycle analysis. Fig. 6A and S10A showed the results of cytometric DNA content distribution of MGC-803 and GES-1 cells, respectively. For MGC-803 cells treated with AuNCs@L-GSH and AuNCs@D-GSH, significant accumulation (about 21.0\% and 29.3\%, respectively) in the $\mathrm{G}_{2} / \mathrm{M}$ phase were observed compared with the untreated control cells (about 19\%). However, the GES-1 cells treated with AuNCs@L-GSH or AuNCs@D-GSH did not show massive increase in $\mathrm{G}_{2} / \mathrm{M}$ accumulation (Supplementary Material: Fig. S9A). Therefore, chiral Au NCs induced $\mathrm{G}_{2} / \mathrm{M}$ phase arrest of MCC-803 cells, and retarded cell proliferation. The difference of cell cycle arrest in $\mathrm{G}_{2} / \mathrm{M}$ phase between AuNCs@L-GSH and AuNCs@D-GSH was consistent with the results of cytotoxicity, ROS induction and DNA damage. Our study further confirmed the recognition of ROS as essential players in cells cycle progression. When the intracellular redox homeostasis was interrupted, the functions and activities of several key cell cycle components such as cyclins (i.e. cyclin D1 and E), cyclin dependent kinases (i.e. CDK2 and 4) and cell cycle check point proteins (i.e. Chk1, Chk2) were influenced, which in turn direct the cell to be arrested or ultimately lead to apoptosis [29].

Further, the death mechanisms of MGC-803 and GES-1 cells treated with AuNCs@L-GSH and AuNCs@D-GSH were evaluated and compared by annexin V/PI double staining method. The percentage of cells in early apoptotic, late apoptotic and necrotic stages in MGC-803 cells exposed to $100 \mu \mathrm{g} / \mathrm{ml}$ of AuNCs@L-GSH and AuNCs@D-GSH for $24 \mathrm{~h}$ was quantified by flow cytometry. The data from the annexin V/PI staining experiment indicated that a ma- 
jority of cells was undergoing apoptosis $(13.9 \%$ for early apoptosis, $48.2 \%$ for late apoptosis) rather than necrosis when exposed to AuNCs@D-GSH, while $13.0 \%$ and $3.23 \%$ of cell death observed was due to early and late apoptosis when exposed to AuNCs@L-GSH (Fig. 6B). This was consistent with a higher concentration of intracellular ROS and lower cell viability of MGC-803 cells treated with AuNCs@D-GSH than those treated with AuNCs@L-GSH. Similar results was demonstrated in GES-1 cells after exposure to AuNCs@L-GSH and AuNCs@D-GSH, however, the ratio of cell apoptosis was comparatively lower than that in MGC-803 cells under the same condition (Fig. S9B). The apoptosis assay revealed that induction of apoptosis was associated with increased generation of ROS, reduced mitochondrial membrane potential and cell cycle arrest at $\mathrm{G}_{2} / \mathrm{M}$ phase. In conclusion, AuNCs@D-GSH caused severer cytotoxicity against MGC-803 cells in accordance with the results of above-mentioned MTT assay.

\section{Global gene expression changes with microarray}

\section{Gene expression profiling}

Understanding molecular mechanism underlying the cellular events of a toxicological outcome allows the prediction of molecular pathways in the response of the organism to a stressor [31]. Therefore, global gene expression analysis was employed to comprehend the underlying molecular mechanism of cytotoxicity when exposed to AuNCs@D-GSH/AuNCs@L-GSH. As shown in Fig. $7 \mathrm{~A}$, a total number of 778 genes, including the 317 up-regulated and the 461 down-regulated, were significantly affected by AuNCs@D-GSH and, correspondingly, a total of 405 genes, including the 187 up-regulated and the 218 down-regulated, by AuNCs@L-GSH. Comparatively, the number of genes affected by AuNCs@L-GSH was approximately $1 / 2$ of those by AuNCs@D-GSH. The numbers of differentially expressed genes (DEGs) shared by the Au NCs treatment were represented in the vein diagrams (Fig. 7B). To distinguish the effects of AuNCs@L-GSH and AuNCs@D-GSH on the related biological process of MGC-803 cells, the differentially up- and down-regulated genes were functionally analyzed on the basis of gene ontology (GO).
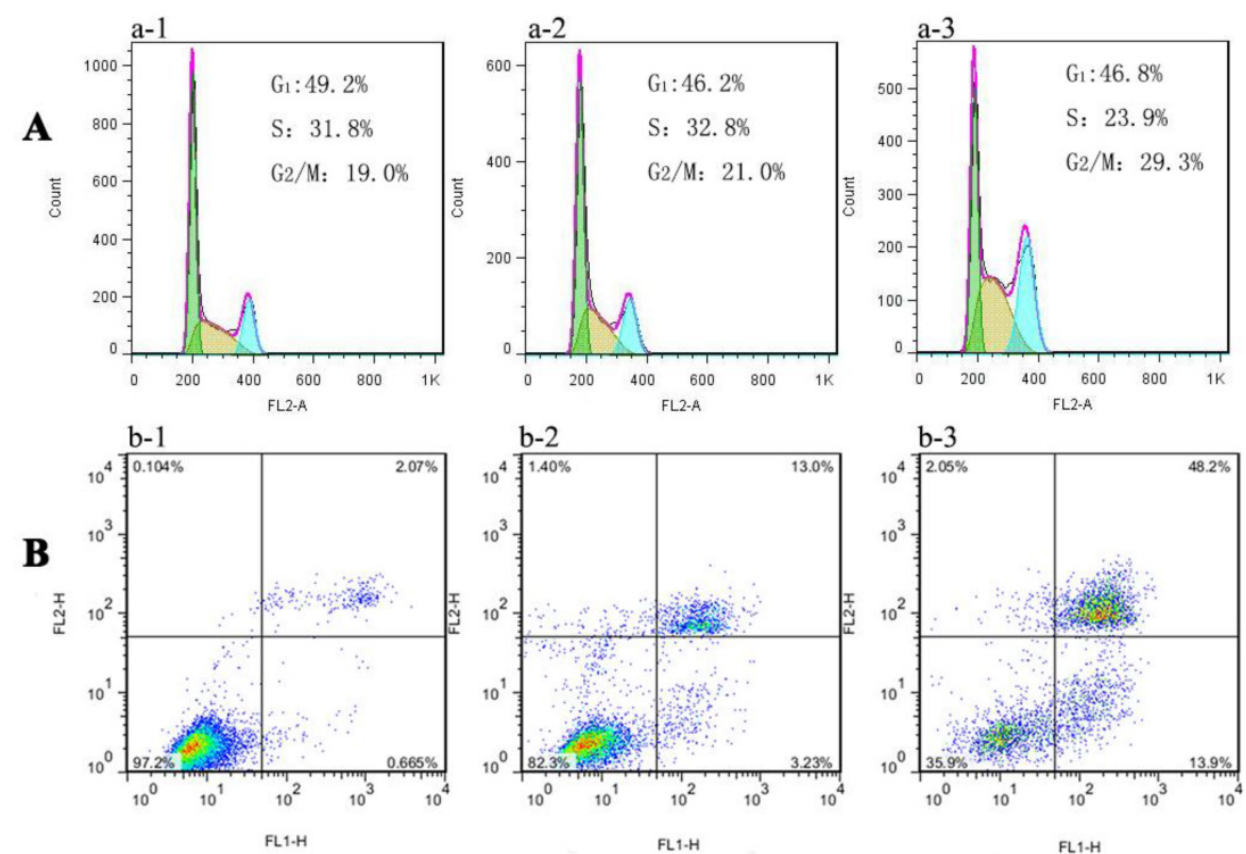

Figure 6. Flow cytometry analysis of (A) cell cycle phase distribution and (B) apoptosis/necrosis in MGC-803 cells after exposure to (a-2, b-2) 100 Mg/ml AuNCs@L-GSH and (a-3, b-3) $100 \mu \mathrm{g} / \mathrm{ml}$ AuNCs@D-GSH for 24 h, respectively. (a-l, b-l) Cells without any treatment. 


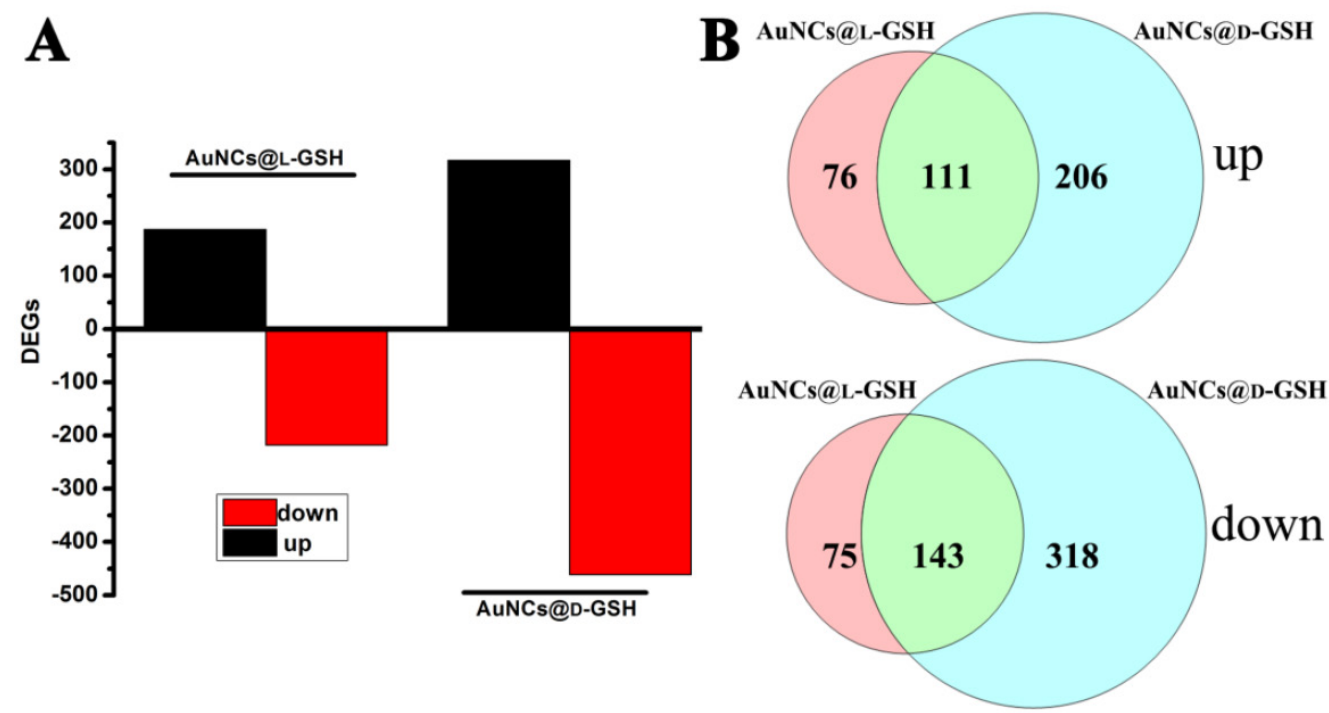

Figure 7. (A) Numbers of genes differentially expressed in MGC-803 cells treated with AuNCs@L-GSH and AuNCs@D-GSH. (B) Venn diagrams with the combinations indicating numbers of shared genes between treatment of AuNCs@L-GSH and AuNCs@D-GSH.

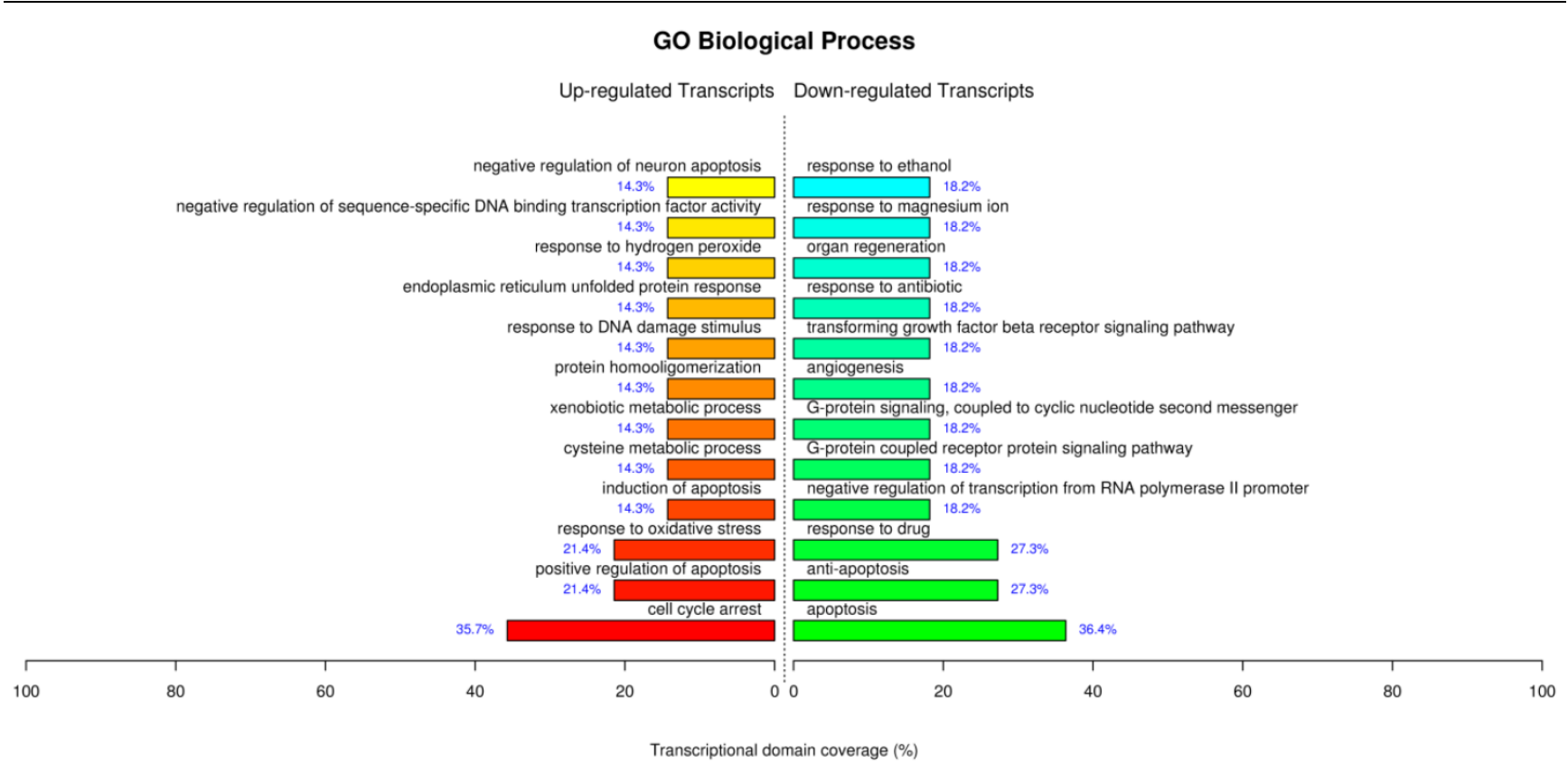

Figure 8. GO terms of biological process significantly affected by AuNCs@L-GSH.

The GO analysis for the treatment of AuNCs@L-GSH by the term biological process showed similar processes which have been supported by our parallel experiments such as cell cycle arrest, positive regulation of apoptosis, response to oxidative stress, response to DNA damage stimulus etc (Fig. 8). For the term molecular function displayed sequence-specific DNA binding transcription factor activity, sequence-specific DNA binding, cystathionine beta and gama-lyase activity, L-cysteine desulfhydrase activity, aldo-keto reductase (NADP) activity etc. Of note, the up-regulated L-cysteine desulfhydrase, which catalyzes the decomposition of L-cysteine to pyruvate, ammonia, and $\mathrm{H}_{2} \mathrm{~S}$, could be specifically responsible for the cysteine degradation leading to the reduced intracellular L-cysteine concentrations and therefore perturb the intracellular redox-homeostatic buffering [32]. Cystathionine gamma-lyase is the rate-limiting enzyme for the synthesis of cysteine and availability of cysteine is a critical factor in glutathione synthesis [33]. Therefore, the up-regulated cystathionine gamma-lyase leads to the production of glutathione and maintaining the redox homeostasis. Aldo-keto reductase (NADP), which primarily reduces aldehydes and ketones to primary and secondary alcohols, plays a central role in the cellular response to osmotic, electrophilic, and oxidative stress [34]. Overall, the GO analysis for AuNCs@L-GSH treatment further cor- 
roborated our perceptions of the molecular mechanism of cytotoxicity. MGC-803 cells treated with AuNCs@D-GSH showed a very different gene expression compared with those treated with AuNCs@L-GSH (Supplementary Material: Fig. S10). AuNCs@D-GSH induced apoptosis-related GO terms up-regulated in like manner, and more significantly, AuNCs@D-GSH individually up-regulated GO terms related with metabolic and biosynthetic process, indicating a synergetic effect in the cytotoxicity of AuNCs@D-GSH. Moreover, Au NCs inhibited cell growth and shifted the balance between pro- and anti-apoptotic genes in favor of apoptosis.

\section{KEGG pathway analysis}

The Kyoto Encyclopedia of Genes and Genomes (KEGG) pathway mapping is a general analysis method to study biological interpretation of high-level systemic functions based on large-scale datasets in genomics, transcriptomics, proteomics, and metabolomics [35,36]. As shown in Fig. 9A, AuNCs@L-GSH affected the largest number of pathways including mineral absorption, p53 signaling pathway, and selenocompound metabolism. AuNCs@D-GSH exposure caused the most statistically significant pathway changes in metabolic pathways and steroid biosynthesis, metabolism of xenobiotics by cytochrome P450 and PPAR signaling pathway were also the enriched pathways.

The difference in the specific genes with altered expression upon the exposure to the two different $\mathrm{Au}$ NCs suggested the different toxic mechanisms. We observed that AuNCs@L-GSH with a relative lower cytotoxicity tending to induce more chemical-specific toxicity response, while AuNCs@D-GSH, which was much more toxic than AuNCs@L-GSH, dominated more metabolic- and biosynthetic-related response (Fig. 9B). In summary, for the first time, our results revealed more detailed transcriptional information on the toxic mechanism of AuNCs@L-GSH and AuNCs@D-GSH. In general, global gene expression analysis in cancer cells in response to nanomaterials promised a revolutionary new ground for monitoring and identifying the biological process and pathways responsible for toxicity, revealing the toxic mechanism and obtaining toxicant-specific molecular fingerprints [37].

\section{GSTP I protects against Au NCs-induced cytotoxicity}

All results above-mentioned showed that different chiral Au NCs demonstrated different toxicity, of which AuNCs@D-GSH exhibited stronger cytotoxicity against MGC-803 cells (Fig. 10A). The potential molecular mechanism was needed to be further clari- fied. We predicted that the inherent hereditary defects of MGC-803 cells may result in the differential cytotoxicity induced by chiral Au NCs. Therefore, we examined the epigenetic difference between MGC803 and GES-1 cells. Epigenetics is the study of reflecting functionally relevant modifications to the genome that do not change the nucleotide sequence, examples of such modifications are DNA methylation and histone modification, which is a bridge between genotype and phenotype [38,39]. DNA methylation often occurs on the cytosine residues in the sequence context 5'-CpG-3', it plays an important role in the control of gene expression in mammalian cells [40]. In different types of tumours, aberrant or accidental methylation of $\mathrm{CpG}$ islands in the promoter region often results in silencing of many cancer-related gene expressions [41]. For example, GSTP1, encoding glutathione S-transferase pi, has been reported to be the target of somatic CpG island hypermethylation in $90 \%$ of prostate cancers, which is recognized as a molecular hallmark of human prostate cancer [42]. The GSTP1 is an important multi-functional detoxifying enzyme within the glutathione S-transferase family enzymes that inactivates electrophilic carcinogens by conjugation with glutathione. Recent findings also confirmed the critical role of GSTP1 in the protection of cells from ROS because they can utilize a wide variety of products of oxidative stress as substrates $[43,44]$.

As shown in Fig. 10B and C, GSTP1 mRNA and protein were not detectable in MGC-803 and MCF-7 cells, while both were present in GES-1 cells. To determine whether the lack of GSTP1 expression in MGC-803 cells was associated with GSTP1 gene methylation, methylation-specific PCR (MSP) was used to explore the methylation status of the $\mathrm{CpG}$ island located at the 5 ' promoter site. The breast cancer MCF-7 cell line, failing to express GSTP1 mRNA or GSTP1 polypeptides due to the hypermethylation of CpG dinucleotides at the 5 transcriptional regulatory region, was chosen as the positive control. As shown in Fig. 10D, abnormal GSTP1 CpG island hypermethylation was detected in MGC-803 cells, but not in GES-1 cells. To determine whether the hypermethylation was associated with GSTP1 transcriptional silencing, MGC-803 cells were treated with 5 and $10 \mu \mathrm{M}$ 5-aza-2'-deoxycytidine (5-aza-dC) for $72 \mathrm{~h}$ respectively. 5-aza-dC is a nucleoside-analogue inhibitor of DNA methyltransferases, which can reactivate epigenetically silenced genes and restore normal gene function [45]. Fig. 10E and F showed that GSTP1 expression was restored after 5-aza-dC treatment. Therefore, we considered that $\mathrm{CpG}$ island hypermethylation was responsible for the lack of GSTP1 expression in the MGC-803 cell line. Then, the effects 
of restoration of GSTP1 expression in MGC-803 cells on the survival rate of cells treated with chiral Au NCs were investigated using MTT assay. As shown in Fig. 10F, the cytotoxicity of AuNCs@D-GSH against MGC-803 cells was markedly diminished. Taken together, all these results highly suggested that GSTP1 protecting GES-1 cells and GSTP1 expression restored MGC-803 cells against oxidative stress caused by chiral $\mathrm{Au}$ NCs and contributing to cell survival. Schematic representation of the cytotoxicity induced by AuNCs@L-GSH and AuNCs@D-GSH was shown in Fig. 11.

\section{Conclusion}

In summary, we firstly report that both AuNCs@L-GSH and AuNCs@D-GSH are capable of inducing ROS generation and activating integrated cytotoxic effects that include mitochondrial depolarization, DNA damage, cell cycle arrest and apoptosis mediated death. Of particular interest is that the D-GSH-capped Au NCs are comparatively more toxic than L-GSH-capped ones for both MGC-803 and GES-1 cells based on the principle of ROS induction.
The cell physiology-based toxicity study demonstrates that the chirality of Au NCs is a crucial feature that determines the type and magnitude of the cellular response. We exploit the technology of microarray to analyze the transcriptome, to obtain comprehensive knowledge of molecular mechanism of the cytotoxic effects. Our data suggest that both L-and D-GSH-capped Au NCs caused the up-regulation of genes involved in cellular rescue and stress response, while AuNCs@D-GSH, affecting MGC-803 cells in a more complex way, individually induced up-regulation of transcripts involved in some metabolic- and biosynthetic-related response. Moreover, MGC-803 cells are more sensitive to the oxidative stress damage induced by Au NCs than GES-1 cells, which may be associated with GSTP1 hypermethylation. This chirality-associated regulation of cytotoxicity highlights the importance of the interplays between chiral materials and biological system at sub-nano level. We expect that a better understanding of mechanism of chiral Au NCs injury, together with epigenetic changes of biological systems, will allow us to predict the modes-of-action of similar agents.

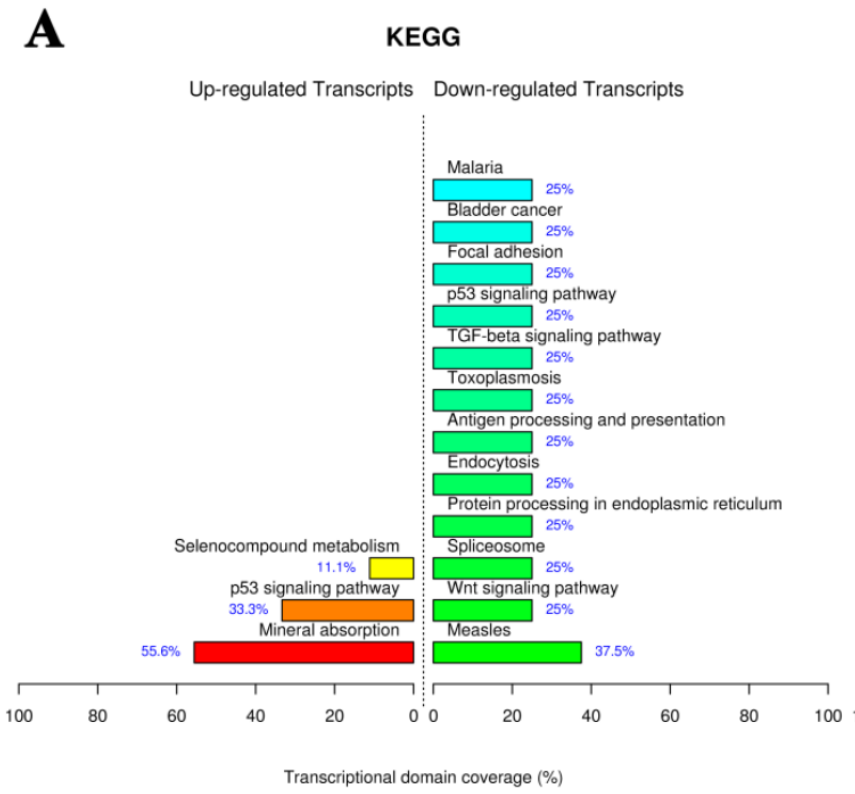

\section{B}

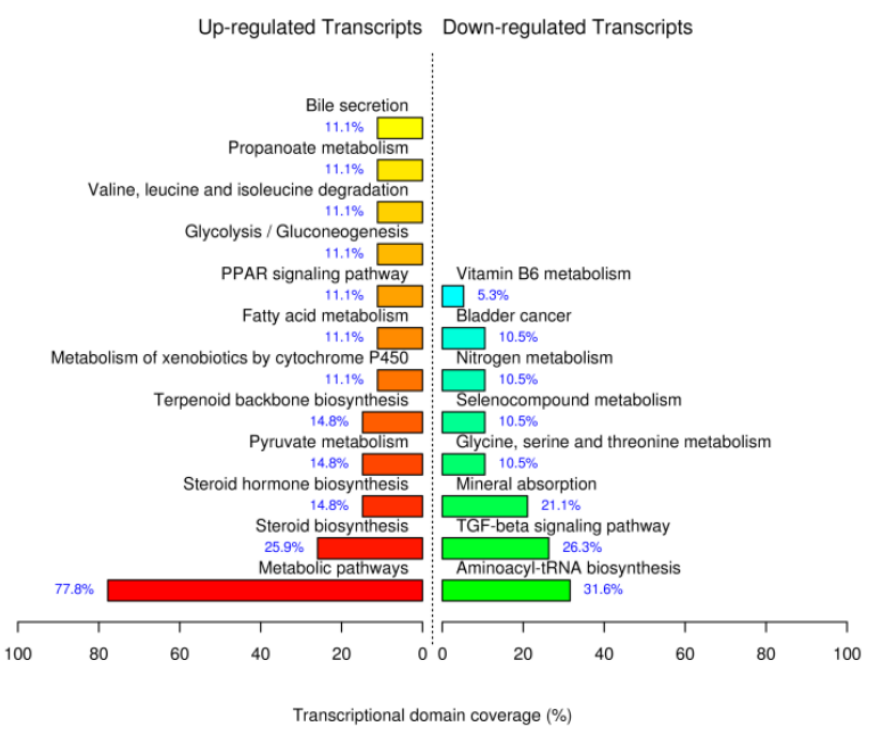

Figure 9. KEGG pathway analysis of gene expression data from MGC-803 cells exposed to (A) AuNCs@L-GSH and (B) AuNCs@D-GSH. Functional profiling depicts significantly overrepresented KEGG pathways on the basis of differentially expressed genes in AuNCs@L-GSH vs. control and AuNCs@D-GSH vs. control. Functional profiling was conducted via the FunNet tool by considering all differentially expresses genes at an FDR (false discovery rate) of $5 \%$. 


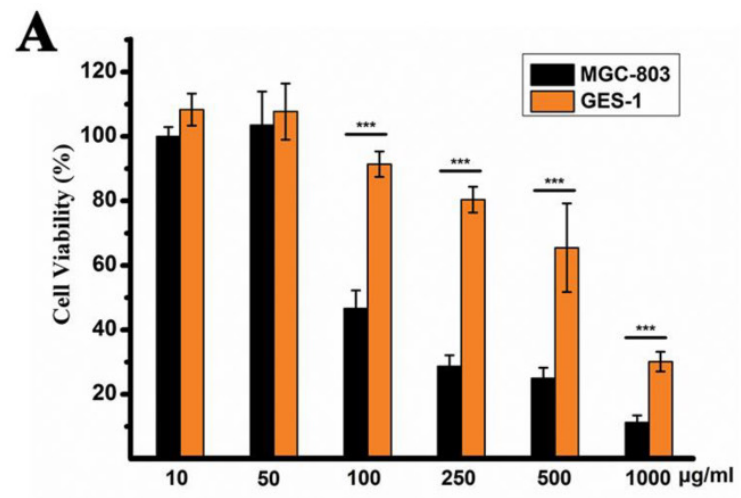

D
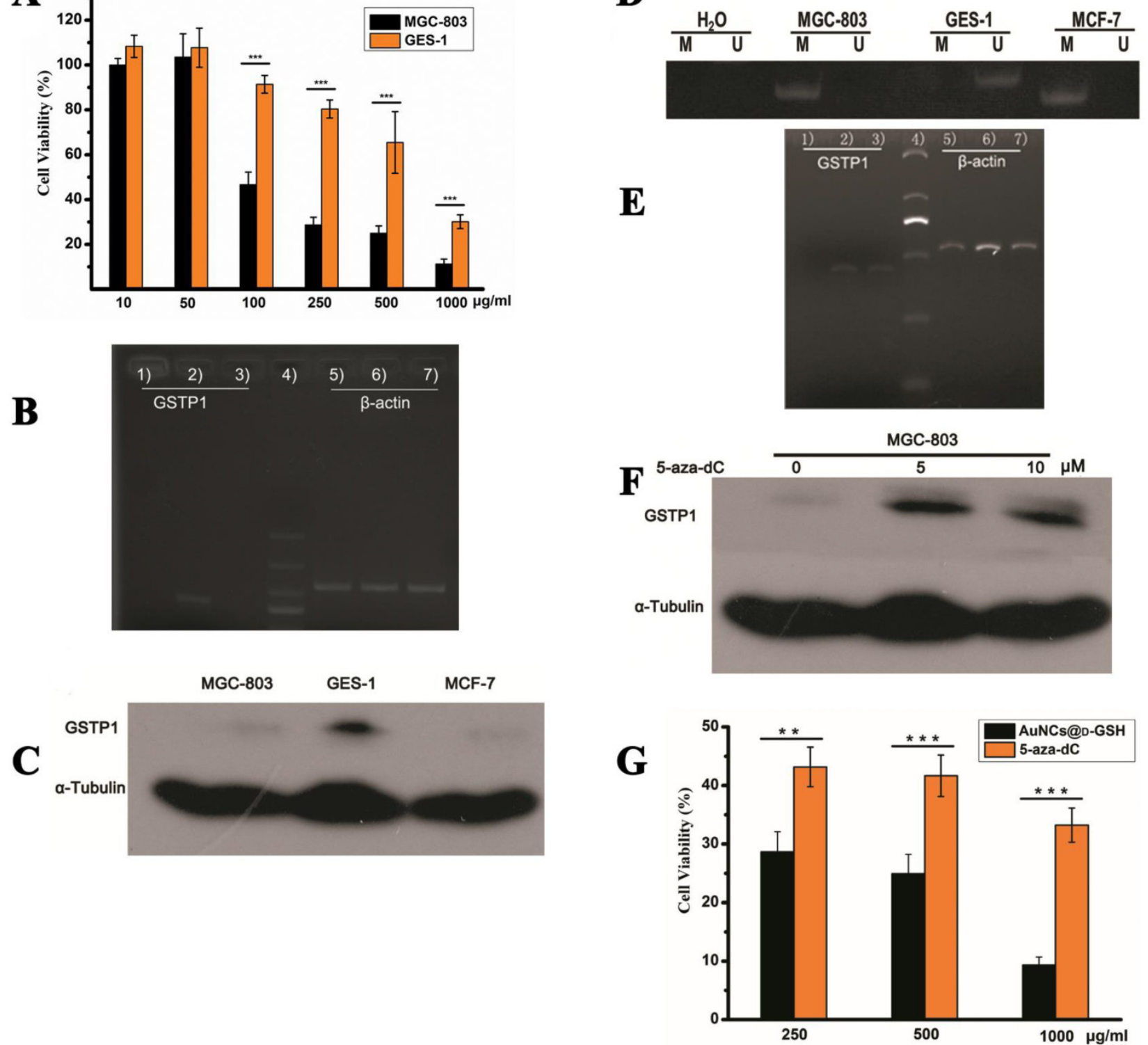

Figure 10. Au NCs-induced cytotoxicity is cell-type-dependent. (A) Cell viability of MGC-803 and GES-I cells after co-incubation with AuNCs@D-GSH for 24 h. (B) GSTPI mRNA levels determined by RT-PCR and agarose gel electrophoresis. I) MGC-803, 2) GES-I, 3) MCF-7, 4) size markers, 6-9) $\beta$-actin internal controls, respectively, from MGC-803, GES-I and MCF-7 cells. (C) GSTPI protein expression in MGC-803, GES-I and MCF-7 cells, tubulin provided the loading control. (D) Methylation status of GSTPI $C_{P G}$ island in different cell lines revealed by MSP. The presence of a PCR product in lanes $M$ indicated that the GSTPI gene is methylated, whereas the product in lanes $U$ indicated that the promoter region is unmethylated. The water control does not show evidence of contamination. M, methylated; $U$, unmethylated. (E) The restoration of GSTPI mRNA was determined by RT-PCR and agarose gel electrophoresis. I, 2) and 3) were MGC-803 cells and the cells treated with $5 \mu M$ and I0 $\mu$ M 5 -aza-dC for 72 h, respectively. (F) The restoration of GSTPI protein expression in MGC-803 cells was detected by western blotting after treatment with 5 and $10 \mu M$-aza-dC for 72 h, respectively. (G) Effect of 5 -aza-dC treatment on the cell viability of MGC-803 cells when co-incubation with AuNCs@D-GSH. **p<0.0I, *** $p<0.00$ I, Student's $t$-test results at $95 \%$ confidence level were significantly different. 


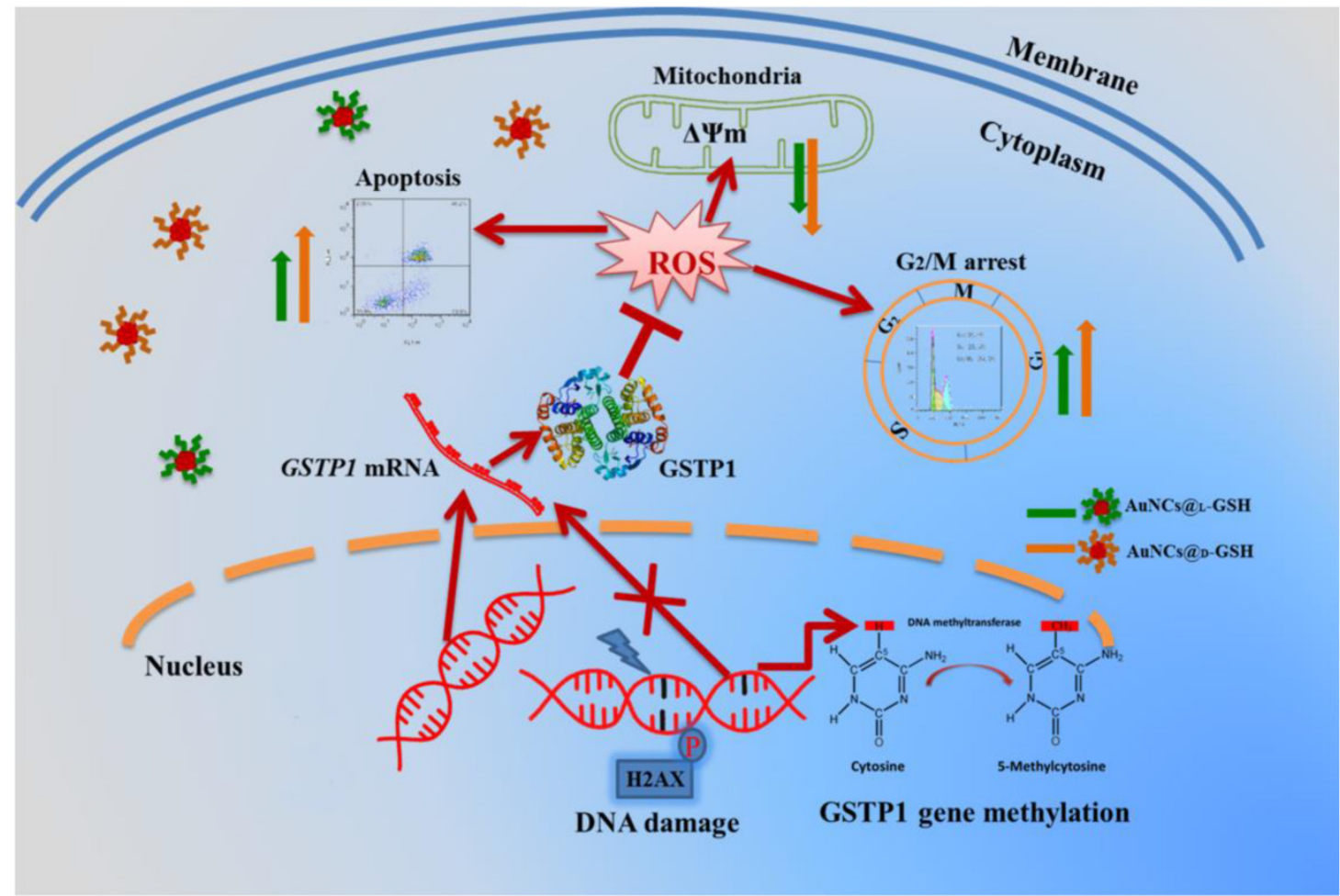

Figure II. Schematic representation of the cytotoxicity induced by AuNCs@L-GSH and AuNCs@D-GSH.

\section{Supplementary Material}

Figs. S1 - S10, Table S1.

http://www.thno.org/v05p0134s1.pdf

\section{Acknowledgements}

This work is supported by National Key Basic Research Program (973 Project) (No. 2010CB933901), the National Natural Scientific Foundation of China (Grant No. 81225010, 20803040, 81028009, and 31170961).

\section{Competing Interests}

The authors report no conflicts of interest.

\section{References}

1. Blackmond DG. The origin of biological homochirality. Cold Spring Harbor perspectives in biology. 2010; 2: a002147.

2. Amabilino D. Chirality at the Nanoscale: Nanoparticles, surfaces, materials and more. Germany: Wiley-VCH Weinheim Publisher; 2009.

3. Farrag M, Tschurl M, Heiz U. Chiral gold and silver nanoclusters: Preparation, size selection, and chiroptical properties. Chem Mater. 2013; 25: 862-70.

4. Slocik JM, Govorov AO, Naik RR. Plasmonic circular dichroism of peptide-functionalized gold nanoparticles. Nano lett. 2011; 11: 701-5.

5. Zhu M, Qian H, Meng X, Jin S, Wu Z, Jin R. Chiral Au 25 nanospheres and nanorods: synthesis and insight into the origin of chirality. Nano lett. 2011; 11: $3963-9$

6. Zhang C, Zhou Z, Qian Q, Gao G, Li C, Feng L, et al. Glutathione-capped fluorescent gold nanoclusters for dual-modal fluorescence/X-ray computed tomography imaging. J Mater Chem B. 2013; 1: 5045-53.

7. Qian H, Zhu M, Wu Z, Jin R. Quantum sized gold nanoclusters with atomic precision. Acc Chem Res. 2012; 45: 1470-9.

8. Xu Q, Kumar S, Jin S, Qian H, Zhu M, Jin R. Chiral 38-Gold-Atom Nanoclusters: Synthesis and Chiroptical Properties. Small. 2013.

9. Knoppe S, Bürgi T. Chirality in Thiolate-Protected Gold Clusters. Acc Chem Res. 2014; 47: 1318-26.
10. Schaaff TG, Knight G, Shafigullin MN, Borkman RF, Whetten RL. Isolation and selected properties of a $10.4 \mathrm{kDa}$ gold: glutathione cluster compound. J Phys Chem B. 1998; 102: 10643-6.

11. Yao H, Fukui T, Kimura K. Chiroptical responses of D-/L-penicillamine-capped gold clusters under perturbations of temperature change and phase transfer. J Phys Chem C. 2007; 111: 14968-76.

12. Wu G, Fang Y-Z, Yang S, Lupton JR, Turner ND. Glutathione metabolism and its implications for health. J Nutr. 2004; 134: 489-92.

13. Knoppe S, Dolamic I, Bürgi T. Racemization of a chiral nanoparticle evidences the flexibility of the gold-thiolate interface. J Am Chem Soc. 2012; 134: 13114-20.

14. Dolamic I, Knoppe S, Dass A, Bürgi T. First enantioseparation and circular dichroism spectra of $\mathrm{Au}_{38}$ clusters protected by achiral ligands. Nat Commun. 2012; 3: 798-804

15. Wang Y, Chen J, Irudayaraj J. Nuclear targeting dynamics of gold nanoclusters for enhanced therapy of HER2+ breast cancer. ACS nano. 2011; 5: 9718-25.

16. Liu J, Yu M, Zhou C, Yang S, Ning X, Zheng J. Passive tumor targeting of renal-clearable luminescent gold nanoparticles: long tumor retention and fast normal tissue clearance. J Am Chem Soc. 2013; 135: 4978-81.

17. Zhang X-D, Luo $Z$, Chen J, Shen $X$, Song $S$, Sun $Y$, et al. Ultrasmall $\mathrm{Au}_{10-12}(\mathrm{SG})_{10-12}$ Nanomolecules for High Tumor Specificity and Cancer Radiotherapy. Adv Mater. 2014; 26: 4565-8.

18. Zhang X-D, Chen J, Luo Z, Wu D, Shen X, Song S-S, et al. Enhanced Tumor Accumulation of Sub-2 nm Gold Nanoclusters for Cancer Radiation Therapy. Adv Healthc Mater. 2014; 3: 133-41

19. Li Y, Zhou Y, Wang HY, Perrett S, Zhao Y, Tang Z, et al. Chirality of glutathione surface coating affects the cytotoxicity of quantum dots. Angew Chem Int Ed. 2011; 50: 5860-4.

20. LeBel CP, Ischiropoulos $\mathrm{H}$, Bondy SC. Evaluation of the probe 2', 7 -dichlorofluorescin as an indicator of reactive oxygen species formation and oxidative stress. Chem Res Toxicol. 1992; 5: 227-31.

21. Pastore A, Federici G, Bertini E, Piemonte F. Analysis of glutathione: implication in redox and detoxification. Clin Chim Acta. 2003; 333: 19-39.

22. Pan Y, Leifert A, Ruau D, Neuss S, Bornemann J, Schmid G, et al. Gold nanoparticles of diameter $1.4 \mathrm{~nm}$ trigger necrosis by oxidative stress and mitochondrial damage. Small. 2009; 5: 2067-76.

23. Ly J, Grubb D, Lawen A. The mitochondrial membrane potential $(\Delta \Psi \mathrm{m})$ in apoptosis; an update. Apoptosis. 2003; 8: 115-28.

24. Kroemer G, Reed JC. Mitochondrial control of cell death. Nat Med. 2000; 6: 513-9.

25. Karna P, Zughaier S, Pannu V, Simmons R, Narayan S, Aneja R. Induction of reactive oxygen species-mediated autophagy by a novel microtubule-modulating agent. J Biol Chem. 2010; 285: 18737-48.

26. Mah L, El-Osta A, Karagiannis T. $\gamma \mathrm{H} 2 \mathrm{AX}$ : a sensitive molecular marker of DNA damage and repair. Leukemia. 2010; 24: 679-86. 
27. AshaRani P, Low Kah Mun G, Hande MP, Valiyaveettil S. Cytotoxicity and genotoxicity of silver nanoparticles in human cells. ACS nano. 2008; 3: 279-90.

28. Tanaka H, Arakawa H, Yamaguchi T, Shiraishi K, Fukuda S, Matsui K, et al. A ribonucleotide reductase gene involved in a p53-dependent cell-cycle checkpoint for DNA damage. Nature. 2000; 404: 42-9.

29. Fan LM, Li J-M. Evaluation of methods of detecting cell reactive oxygen species production for drug screening and cell cycle studies. J Pharmacol Toxicol Methods. 2014; 70: 40-7.

30. Zhou YB, Feng X, Wang LN, Du JQ, Zhou YY, Yu HP, et al. LGH00031, a novel ortho-quinonoid inhibitor of cell division cycle 25B, inhibits human cancer cells via ROS generation. Acta Pharmacol Sin. 2009; 30: 1359-68.

31. Pillai S, Behra R, Nestler H, Suter MJ-F, Sigg L, Schirmer K. Linking toxicity and adaptive responses across the transcriptome, proteome, and phenotype of Chlamydomonas reinhardtii exposed to silver. Proc Natl Acad Sci. 2014; 111: 3490-5.

32. Xie Y, Lai D, Mao Y, Zhang W, Shen W, Guan R. Molecular cloning, characterization, and expression analysis of a novel gene encoding L-cysteine desulfhydrase from Brassica napus. Mol Biotechnol. 2013; 54: 737-46.

33. Diwakar L, Ravindranath V. Inhibition of cystathionine-gamma-lyase leads to loss of glutathione and aggravation of mitochondrial dysfunction mediated by excitatory amino acid in the CNS. Neurochem Int. 2007; 50: 418-26.

34. Jin Y, Penning TM. Aldo-keto reductases and bioactivation/detoxication. Annu Rev Pharmacol Toxicol. 2007; 47: 263-92.

35. Chatterjee N, Eom HJ, Choi J. A systems toxicology approach to the surface functionality control of graphene-cell interactions. Biomaterials. 2014; 35: 1109-27.

36. $\mathrm{Li} \mathrm{X}, \mathrm{He} \mathrm{Q}$, Shi J. Global gene expression analysis of cellular death mechanisms induced by mesoporous silica nanoparticle-based drug delivery system. ACS Nano. 2014; 8: 1309-20.

37. Gou N, Onnis-Hayden A, Gu AZ. Mechanistic toxicity assessment of nanomaterials by whole-cell-array stress genes expression analysis. Environ Sci Technol. 2010; 44: 5964-70.

38. Eichten SR, Schmitz RJ, Springer N. Epigenetics: more than chromatin modifications and complex gene regulatory systems. Plant Physiol. 2014.

39. Jones PA, Takai D. The role of DNA methylation in mammalian epigenetics. Science. 2001; 293: 1068-70.

40. Ronneberg JA, Tost J, Solvang HK, Alnaes GI, Johansen FE, Brendeford EM, et al. GSTP1 promoter haplotypes affect DNA methylation levels and promoter activity in breast carcinomas. Cancer Res. 2008; 68: 5562-71.

41. Ehrlich M. DNA methylation in cancer: too much, but also too little. Oncogene. 2002; 21: 5400-13.

42. Cairns $\mathrm{P}$, Esteller M, Herman JG, Schoenberg M, Jeronimo C, Sanchez-Cespedes M, et al. Molecular detection of prostate cancer in urine by GSTP1 hypermethylation. Clin Cancer Res. 2001; 7: 2727-30.

43. Hayes JD, Strange RC. Potential contribution of the glutathione S-transferase supergene family to resistance to oxidative stress. Free Radic Res. 1995; 22: 193-207.

44. Kumar V, Yadav CS, Datta SK, Singh S, Ahmed RS, Goel S, et al. Association of GSTM1 and GSTT1 polymorphism with lipid peroxidation in benign prostate hyperplasia and prostate cancer: a pilot study. Dis Markers. 2011; 30: 163-9.

45. Christman JK. 5-Azacytidine and 5-aza-2'-deoxycytidine as inhibitors of DNA methylation: mechanistic studies and their implications for cancer therapy. Oncogene. 2002; 21: 5483-95.

46. Prifti E, Zucker J-D, Clement K, Henegar C. FunNet: an integrative tool for exploring transcriptional interactions. Bioinformatics. 2008; 24: 2636-8. 